\title{
Raum erfahren
}

\author{
Epistemologische, ethische und ästhetische Zugänge \\ Hrsg. v. David Espinet, Tobias Keiling u. Nikola Mirkovic
}

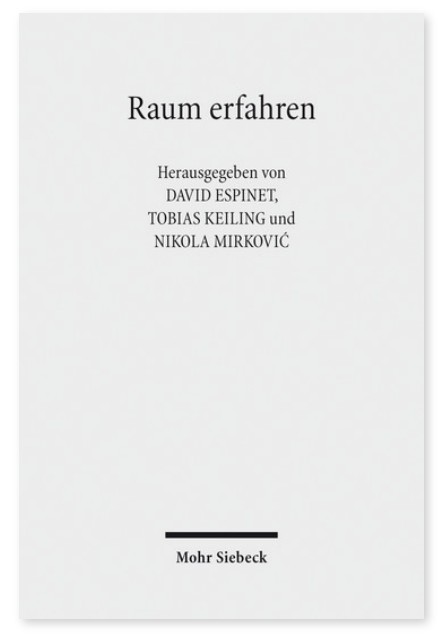

2017. VIII, 250 Seiten.

ISBN 978-3-16-154963-2 DOI 10.1628/978-3-16-154963-2 eBook PDF 69,00€

ISBN 978-3-16-154931-1 fadengeheftete Broschur 69,00€
Der Raum ist ein philosophisches Thema par excellence. Denn so selbstverständlich es ist, dass wir in Räumen und im Raum leben, so unklar ist, was das bedeutet. Wie verhalten sich lebensweltliche Räume zu 'dem Raum' überhaupt? Gibt es bevorzugte Formen der Raumerfahrung? Wie verhalten sich Raum und Zeit zueinander? Was unterscheidet Nähe und Distanz, Bewegung und Aufenthalt?

Die Beiträge des vorliegenden Bandes nähern sich der Philosophie des Raumes aus Richtung der Epistemologie, praktischen Philosophie und Ästhetik. Dahinter steht die Überzeugung, dass der Raum nicht allein ein Thema der theoretischen Philosophie sein sollte, sondern Raumphänomene in allen Bereichen der Philosophie relevant sind. Im Zusammenspiel von klassischen und gegenwärtigen Positionen ergibt sich ein Überblick über die Möglichkeit, eines der unscheinbarsten Phänomene überhaupt zu thematisieren.

Inhaltsübersicht

John Sallis: Über Grenzen und Weite des enchorialen Raums - Markus Gabriel: Raum und Feld - Alexander Schnell: Marc Richirs Phänomenologie der Raumkonstitution - Diego D'Angelo: Transzendentaler Abstand und Interpretation in Husserls Ding und Raum - Michael N. Forster: Kants transzendentaler Idealismus. Das Argument hinsichtlich das Raumes und der Geometrie - Tobias Keiling: Logische und andere Räume. Wittgenstein und Blumenberg über Unbestimmtheit - Georg Bertram: Die konstitutive Räumlichkeit ästhetischer Erfahrung - Nikola Mirković: Zur Räumlichkeit der Musik - Steven G. Crowell: Interiors. The space of meaning and the Great Indoors - David Espinet: Raumgebende Zeit? Zur empirischen, historischen und ästhetischen Individuation des Raumes - Inga Römer: Spatium-Topos-Atopos. Zur Phänomenologie des Raumes - Ole Meinefeld: Die Enthauptung Ludwig XVI. 1793 im Spiegel von Claude Leforts Demokratietheorie - Christian Bermes: Tatort und Handlung - Jocelyn Benoist: Space as Achieved Impossibility - Volker Gerhardt: Die Apriorität des Raumes

David Espinet ist Gastprofessor für Philosophie an der Universität Ulm und Privatdozent an der Albert-Ludwigs-Universität Freiburg im Breisgau.

Tobias Keiling ist wissenschaftlicher Mitarbeiter am Institut für Philosophie der Universität Bonn; 2019/2020 Feodor LynenForschungsstipendiat der Alexander von Humboldt-Stiftung am Somerville College der Universität Oxford. https://orcid.org/0000-0001-6076-8431

Nikola Mirković Geboren 1983; Studium der Philosophie, Psychologie und Slavistik in Freiburg, Basel und Moskau; Forschungsaufenthalt am Philosophy Department des Boston College (USA); 2014 Promotion; Wissenschaftlicher Mitarbeiter am Philosophischen Seminar der Albert-Ludwigs-Universität Freiburg, seit 2016 Wissenschaftlicher Mitarbeiter am Institut für Philosophie der Universität Koblenz-Landau.

Jetzt bestellen:

https://mohrsiebeck.com/buch/raum-erfahren-9783161549632?no_cache=1

order@mohrsiebeck.com

Telefon: +49 (0)7071-923-17

Telefax: +49(0)7071-51104 